\title{
Germanica
}

\section{«Hasta la muerte» Der Sieg der Häßlichkeit in Feuchtwangers Roman Goya}

"Hasta la Muerte ". La victoire de la laideur dans le roman de Feuchtwangers Goya

\section{Frédéric Weinmann}

\section{OpenEdition}

\section{Journals}

Édition électronique

URL : http://journals.openedition.org/germanica/462

DOI : $10.4000 /$ germanica.462

ISSN : 2107-0784

\section{Éditeur}

Université de Lille

\section{Édition imprimée}

Date de publication : 1 décembre 2005

Pagination : 29-39

ISBN : 2-913857-16-7

ISSN : 0984-2632

\section{Référence électronique}

Frédéric Weinmann, « «Hasta la muerte» Der Sieg der Häßlichkeit in Feuchtwangers Roman Goya », Germanica [Online], 37 | 2005, Online erschienen am: 07 Januar 2010, abgerufen am 06 Oktober 2020 URL : http://journals.openedition.org/germanica/462 ; DOI : https://doi.org/10.4000/germanica.462

Ce document a été généré automatiquement le 6 octobre 2020.

(c) Tous droits réservés 


\section{«Hasta la muerte» Der Sieg der Häßlichkeit in Feuchtwangers Roman Goya}

" Hasta la Muerte ». La victoire de la laideur dans le roman de Feuchtwangers Goya

Frédéric Weinmann

\section{Für Ute Kundrus}

1 Goya oder Der arge Weg der Erkenntnis, 1951 erschienen, ist einer der letzten Romane Feuchtwangers. Zusammen mit Die Füchse im Weinberg (ursprünglich Waffen für Amerika betitelt) und Narrenweisheit oder Tod und Verklärung des Jean-Jacques Rousseau bildet er eine Trilogie, in der die französische Revolution als Sinnbild für eine Zeit des Umbruchs steht. Der Künstlerroman schildert das Leben bzw. die geistige und künstlerische Entwicklung Francisco de Goyas zwischen 1793 und 1803. Wie es der letzte Satz des Buches erklärt, hätte diese Romanbiographie eigentlich ein erster Teil werden sollen - der zweite wurde aber nie geschrieben.

2 Auch wenn der Autor berühmt ist für die Fülle an Informationen, die er im Vorfeld sammelte, pflegt er einen sehr freien Umgang mit der historischen Wirklichkeit. Feuchtwanger verfolgt das Prinzip der Analogie zwischen Vergangenheit und Gegenwart und passt die Chronologie und die Fakten je nach Bedarf an den erzählerischen Aufbau an. So weist seine Inquisition eindeutige Parallelen mit dem McCarthysm auf und sein «Goya» erinnert ebenfalls an manchen Stellen an Picasso. So will er auch im berühmten Gemälde Doña Desnuda Cateyana Alba sehen, welche ganz in der romantischen Tradition als große Leidenschaft des Künstlers konzipiert ist, obwohl nichts Überzeugendes diesen Schluß erlaubt und es sich bei diesem Akt sehr wahrscheinlich eher um Pepa Tudó handelt, die Mätresse des Ministers Godoy, bei dem sich das Bild befand ${ }^{1}$. 
3 Für Feuchtwanger (ganz im Sinne Malraux') ist Goya der erste Maler der Moderne. Der Schriftsteller läßt unmißverständlich eine der Romanfiguren, den Doktor und Kunstsammler Peral, schwärmerisch behaupten: «Diese Bilder [...] sind die ersten Schöpfungen einer neuen Kunst, die ersten Bilder des kommenden Jahrhunderts.» $(\mathrm{S} .224)^{2}$. Die Leistung Goyas bestünde in der Überwindung der klassizistischen Ästhetik, die seinerseits Jacques-Louis David verkörperte, von dem es eingangs in bezug auf Marats Tod heißt: «Wie schön und groß bei aller Realistik sprang das häßliche Gesicht des Toten einen an.» (S. 43)

4 Der Roman bildet einen Rückblick auf den Anfang der Epoche, in der die Kunst nicht mehr alles verschönerte, in der die Kategorie des Schönen dennoch immer noch Gültigkeit hat. Nicht zufällig meint der Titelheld als Entschuldigung für einen unmöglichen Auftrag ironisch und doch treffend: «Ich bin nicht eben ein Maler der Schönheit [...].» (S. 260) Diese Tatsache ist offenbar das, was Feuchtwanger an Goyas Kunst am meisten beeindruckte. Die Einführung des Häßlichen in der bildenden Kunst darf daher als Kernmotiv des Romans bezeichnet werden. Der Schriftsteller thematisiert nicht nur den Kampf zwischen Schönheit und Häßlichkeit, nicht nur die Darstellung der Häßlichkeit, sondern die Rezeption dieser Darstellung, das Verhältnis zum Bild.

5 In seinem fiktiven Spanien des ausgehenden 18. Jahrhunderts ist die Schönheit allgegenwärtig. Im gesamten Roman sind 93 Okkurrenzen des Wortes «schön» zu zählen, wobei es sich allerdings 24 Mal nicht auf das menschliche Aussehen bezieht, sondern auf Gegenstände oder auf «das Schöne» an sich, wenn es nicht gar in Redewendungen wie «schön und gut» (S. 343) vorkommt. Nichtdestotrotz gibt es viele bewundernswerte Figuren - zum Beispiel die Marquesa de Villabrancha, welche «noch immer schön» (S. 12) wirkt, oder die Frau des Ministers Godoy, Doña Teresa, deren Gesicht als «länglich, nicht schön, doch anziehend» (S. 509) bezeichnet wird. Dabei sind drei Frauen von außerordentlicher Schönheit, nämlich die untreue Lucía Bermúdez (8 Mal), die Kurtisane Pepa Tudó (15 Mal) und Cateyana Alba (20 Mal), Erbin des großen Generals, Frau des Herzogs José von Alba, persönliche Feindin der Königin und angebliche Liebhaberin Goyas.

6 Abwechselnd benutzt Feuchtwanger gleichfalls das Adjektiv «hübsch», allerdings etwas weniger oft (insgesamt $46 \mathrm{Mal}$ ) und ohne daß der Unterschied $\mathrm{zu}$ "schön» ausschlaggebend wäre. Zum Teil könnte man meinen, daß «hübsch» bevorzugt in Hinsicht auf junge Leute (die Tochter des französischen Botschafters bzw. den Sohn Goyas zum Beispiel) oder wie im Falle des sechzehnjährigen Infanten Fernando «mit nichtsagendem, leidlich hübschem Gesicht» (S. 323) für zweischneidige Komplimente Verwendung findet. Oft genug werden aber dieselben Figuren bald mit dem einem, bald mit dem anderen Terminus beschrieben. So sieht Lucía Bermúdez hier «damenhaft hübsch» (S. 39) aus und ist dort von «damenhafte(r) Schönheit» (S. 72). Von Manuel Godoy heißt es interessanterweise, er habe ein blasiertes Lächeln «auf dem schönen, etwas schweren Anlitz» (S. 94) und einige Jahre später immer noch ein «hübsche(s), etwas schwere(s) Gesicht» (S. 314).

7 Das letzte Beispiel veranschaulicht die Subjektivität des Urteils über menschliche Schönheit: «Etwas schwer» und dennoch «schön» klingt einigermaßen paradox. Das Gesicht des Favoriten der Königin sei «voll, faul» (S. 53) und habe «etwas sonderbar Starres» (ebd.) - unter diesen Umständen kann man sich wohl fragen, wie es immer noch als «schön» gelten kann. Im Grunde besagt dieses Adjektiv eher wenig über das 
Aussehen der Figuren und muß meist durch weitere Qualifikative ergänzt werden. Dabei mögen extrem andere Eigenschaften schön wirken, ohne daß das Urteil dafür weniger kategorisch ausfällt: So besitze Cateyana Alba «schöne ungenierte Augen» (S. 10), während ihr Mann José im Gegenteil «schöne große nachdenkliche Augen» habe (S. 250).

Wer oder was nicht schön ist, steht genauso fest. Es ist zunächst einmal Goya selbst, dessen Beschreibung am Anfang des Romans mit dem endgültigen Verdikt eingeleitet wird: «Er war nicht mehr jung, fünf-und-vier-zig Jahre alt war er, und er war nicht schön.» (S. 15) Diese knappe Beurteilung ist einer der Grundpfeiler seiner Erscheinung. Es ist offenbar das erste, was ihn kennzeichnet. Wie später seine Taubheit, wirkt diese seine Häßlichkeit sogar beinahe als reizend. Nicht zufällig spielt die letzte Liebkosung seiner Mätresse auf sein Aussehen an: «Ach, du häßlicher und / Einz'ger Mann, wie bist du dumm!» (S. 408 und S. 599)

9 Vor allen anderen verkörpert aber die Königin die Häßlichkeit. Ihr bourbonisches Gesicht ist ein Leitmotiv des Romans. Von 35 Okkurrenzen des Adjektivs «häßlich» beziehen sich 19 auf María Luisa. Dieser verebliche Makel ist charakteristisch für die königliche Familie, deren Mitglieder - wie auf einem berühmten Bild Goyas zu sehen ist - bis auf die unehelichen Kinder abstoßend aussehen. Schlimmer als die Königin ist sogar ihre Schwägerin María Josefa, die als «unsäglich häßlich» (S. 323) abgestempelt wird und von der der Romanautor sogar schreibt: «Die abschreckende Häßlichkeit der alten Infantin faszinierte ihn.» (S. 323)

In dieser Hinsicht ist zu bemerken, daß Feuchtwangers Rekurs auf die Kategorien des Schönen und des Häßlichen durch und durch banal ist. Wie es sich im bürgerlichen Roman gehört ${ }^{3}$, sind die Heldinnen, in die man sich verliebt, bei ihm wie selbstverständlich schön. Diese undifferenzierte Bezeichnung der anziehenden Frauen ist im wahrsten Sinne des Wortes ein Stereotyp. Insbesondere im Falle der Pepa Tudó glaubt man zu erraten, wie der Schriftsteller mit einem bestimmten Porträt ${ }^{4}$ arbeitete, das er verinnerlicht hatte und als Schablone systematisch anwendete. Es ist keine Entwicklung zu beobachten in der Beschreibung der Kurtisane, die insgesamt fünfmal «schön und gelassen dasaß» (S. 34, S. 69, S. 260, S. 504, S. 617).

Dabei würde es an Kitsch grenzen, wenn Feuchtwanger diese grobe Wiederholung doch nicht bewußt einzusetzen schien als Reflex für die Oberflächlichkeit dieser Frauenperson: Pepa ist genauso hübsch wie «die schöne, junge Frau» (S. 35), wie eine der «zweihundert schöne(n) und elegante(n) Madrileninnen, Majas, Petitmetras» (S. 74), wie «Raquel la fermosa, 'die schöne Rahel'» (S. 135) aus den alten sentimentalen Romanzen, die sie, «romantischer Kopf» (S. 622) wie sie ist, immer wieder vorsingt.

12 Nicht weniger plakativ ist die Bedeutung der Häßlichkeit, die als Mangel, Schwäche, Abnormität andere Talente fördern soll. Diese psychologisch einfache Vorstellung ist bei Feuchtwanger tief verankert. Schon in seinem zweiten historischen Roman Die häßliche Herzogin Margarete Maultasch, einer Auftragsarbeit, die er unmittelbar nach dem Jud Süß 1922-23 schrieb, symbolisiert die Protagonistin ebengerade wegen ihrer Häßlichkeit das Moderne. Während ihre Rivalin Agnes von Flavon, die «schöne Andere», selbst im Tod zu triumphieren schien, mußte die Außenseiterin durch geistige Vorzüge ihre äußerliche Benachteiligung auszugleichen versuchen. Exakt dasselbe Prinzip nahm er anschließend im seinem stark durch Brecht beeinflußten Drama Die Petroleuminseln wieder auf, in dem die häßliche Präsidentin eines amerikanischen 
Ölkonzerns von ihrer schönen Widersacherin als «Äffin» beschimpt wird und sich durch ihre Geschäftstüchtigkeit zu behaupten hat.

Im Goya entspricht María Luisa ganz und gar diesem Klischee, das Feuchtwanger am Anfang seiner Karriere schon mehrfach benutzte. Insofern vergleichbar mit der Taubheit des Malers, zwingt die Häßlichkeit die Königin zur Selbstüberwindung, in ihrem Fall also zu machtpolitischer Gier und sexueller Sucht. Nichts Originelles im Kernmotiv, das laut Sternburg von Darwin und Freud stamme ${ }^{5}$. Wie in den Werken aus den Zwanzigern findet im Goya ein Duell statt zwischen der jüngeren Schönen und der alternden Häßlichen, die hier wie dort als Siegerin hervorgehen muß, selbst bevor die Rivalin frühzeitig ums Lebens gekommen ist:

Und doch war es besser, wie es war. Nicht mehr viele Jahre, dann wird zum Beispiel die Alba so alt sein, wie sie heute ist, und was wird sie dann sein, die Alba? Verwelkt, vertan. Sie aber, María Luisa, war durch ihre Häßlichkeit gezwungen gewesen, Verstand zu entwickeln. Sie war gescheit geworden, weil sie häßlich war, und ihre Gescheitheit dauerte fort. (S. 442)

«Gescheit» ist das rechte Wort. Gescheit sein ist die Kompensierung für die Behinderung, der ein unvorteilhaftes Aussehen gleichkommt: «Ja, meinte die Alba, wenn eine Frau so ausschaut, muß sie wenigstens gescheit sein.» (S. 161) Die triviale Reduzierung der ästhetischen Frage von Schönheit und Häßlichkeit auf eine Frauenproblematik nimmt im Goya sogar märchenhafte Züge an. Feuchtwanger scheut sich nicht davor, die historischen Figuren als Hexen darzulegen:

Die alternde María Luisa hatte den schärferen Verstand und unbegrenzte Macht, aber die andere war herzbeklemmend schön. Böse waren sie beide, hexenhaft, und es war die Frage, welche der beiden Hexen die gefährlichere war, die schöne oder die häßliche. (S. 102)

Zum Glück bleibt Feuchtwanger in diesem späteren Werk nicht bei dieser zwischen den Gebrüdern Grimm und Walt Disney einzuordnenden Weltauffassung. Sein Künstlerroman zeugt von einer Faszination für die Darstellung des Häßlichen, die ebenfalls aus den zwanziger Jahren des 20. Jahrhunderts stammt und ihn ein Leben lang begleitete. Bekannterweise führt sein Interesse an Goya auf eine Reise nach Spanien im Jahre 1926 zurück, also auf die Zeit vor dem Exil, was vielleicht auf eine primär ästhetische Anregung hindeutet oder jedenfalls dazu bewegt, die Goya-Lektüre nicht aufs Politische zu beschränken.

Der arge Weg, von dem im Untertitel des Goya die Rede ist, führt zwar unabwendbar am Politischen vorbei, ist dennoch keine rein politische, sondern eine feinere, doppelte Erkenntnis. Der Roman ist nicht so sehr ein Plädoyer für engagierte Kunst als vielmehr eine Überwindung derselben. Am Ende (vgl. S. 649) kreuzen sich in der Tat die beiden Überlegungsstränge, der ästhetische und der politische, der «Hasta la muerte» und der « Ya es Hora», um beide von Feuchtwanger als Schlagwörter ausgesuchten Titel aus den Caprichos aufzunehmen. Anders ausgedrückt zeigen die drei Teile des Romans zwar eine stetige Emanzipation, folgen aber nicht dem einfachen Schema: Vorher, Krise, Nachher. Sie bilden eine Aufeinanderfolge von immer höheren Ebenen, jeder Teil ist an sich eine neue Erkenntnis.

17 Die Erzählung beginnt, als Goya schon 45 ist und endlich begriffen hat, "was Malen heißt» (S. 19). Wenn er frühere Bilder seines Schaffens beurteilt, empfindet er sie als «unecht» (S.138). Sie wirkten steif und künstlich. Auch außenstehende Betrachter erkennen die bahnbrechende Neuheit in den jüngsten Werken. Unter diesem Vorwand lädt ihn sogar seine angehende Mätresse Cateyana Alba ein: «Aber sowie ich zurück bin, 
müssen Sie endlich ein Porträt von mir machen auf Ihre neue Art. Alle Welt schwärmt von Ihren neuen Porträts.» (S. 98)

Über das Klassische hinaus hat er eine neue Form der Schönheit entdeckt, eine neue Farbgebung. Als er zum Beispiel an einem Porträt der Lucía Bermúdez arbeitet, ist er mit dem Ergebnis nicht zufrieden, solange «das Verschwebende, Verwirrende, das Abgründige, worauf es an- (kommt)» (S. 23) nicht vorhanden ist: «Die Tönung war nicht da, auf die es ankam, und was fehlte, war ein Winziges, und was fehlte, war alles.» (S. 23) Erst am nächsten Tag fällt ihm plötzlich ein, wie er sein Ziel erreichen kann. Er muß nur Details ändern und sofort trifft er ohne Mühe den Silberton auf dem gelben Kleid, der den gesamten Eindruck bestimmt.

Am Ende des ersten Teils reicht ihm dieses Können trotzdem nicht mehr. Eine Wende bahnt sich an mit den Skizzen für das Porträt der Königin: «[...] er wollte sie malen, wie sie war: häßlich und interessant.» (S.147) In der zweiten Phase seiner Entwicklung spielt der schöne Schein keine Rolle mehr. Jetzt wird seine Kunst politisch. Zum Teil auf Anregung seiner liberalen Freunde sehnt er sich nach einer «Idioma Universal», die seine Liebhaberin dann in Hinsicht auf das Bild eines Stierkampfes unter der Formel subsumiert: «Ich möchte mittun» (S. 223).

Das große Gemälde jener Phase ist das Bild der Familie Carlos des Vierten, das die Häßlichkeit der Bourbonen kompromißlos bloßstellt und bei Goyas Ehefrau den Kommentar hervorruft: «Gewiß, schön waren die Majestäten auch im Leben nicht, aber auf den Porträts des Raphael Mengs, des Maella, ihres Bruders und selbst auf den früheren Porträts Franciscos waren sie bei aller Ähnlichkeit doch nicht so häßlich.» (S. 339) Auf den Vorwurf der Karikatur hin verteidigt sich dennoch der Künstler mit dem Argument, er sehe seine Modelle nicht hart, sondern wie sie sind (vgl. S. 343). Gerade deswegen ist es für Feuchtwanger ein «politisches Bild» (S. 340), weil Goya die Ordnungsträger mit brutaler Sachlichkeit dargestellt habe.

21 Als ein Meister der traditionellen Romankunst führt Feuchtwanger am Ende des zweiten Teils den Übergang zum dritten ein: Nach einer Krise mit seiner Liebhaberin ist Goya endgültig taub geworden. Im Visuellen eingefangen sieht er nun die Realität wieder einmal ganz anders, er nimmt die Farben besser wahr: «Francisco waren diese Straßen vertraut, und vielleicht sah er jetzt ihre Buntheit schärfer, da ihm der Lärm verstummt war.» (S. 423) Mehrmals kommt der Erzähler im dritten Teil auf die Ertaubung zurück, die im Schaffen des Malers eine neue Dimension, die innere, eröffnete. Seinen Gehilfen fragt Goya zum Beispiel: «Hört man den Schuß knallen? Hört man die Majas flüstern? Merkt man, daß das von einem Tauben gemalt ist?» (S. 486)

Durch eine neuerliche Überwindung seiner bisherigen Erkenntnisse gibt der Maler Farben und Malen auf und konzentriert sich auf Schwarzweißdrucke: Es sind die 80 Caprichos, deren Fratzen beinahe kubistisch mehrere Gesichter gleichzeitig zu zeigen scheinen. Der noch weiser gewordene Goya erblickt jetzt die "wahre Wahrheit» (S. 495): «Erst in diesem letzten Jahr hatte er gelernt, was Leben heißt, was Malen heißt, was Kunst heißt.» (S. 522) In der neuen, ungeheueren, tieferen Welt, die er geschaffen hat, ist nicht nur jegliche Schönheit entschwunden, sondern selbst die Häßlichkeit radikaler geworden - so zum Beispiel beim verstorbenen Freund Martin Zapater, der in Goyas Vision «abgründig häßlich» (S. 566) wirkt: «Es war kein Gesicht mehr, es waren eines Mannes Schamteile.» (ebd.)

Bei diesem besonders scharfen Ausdruck läßt sich in aller Deutlichkeit feststellen, daß der künstleriche Aufstieg in drei Stufen mit einem komplexen Entkleiden zu 
vergleichen ist. Wie oben angedeutet, fängt die Selbstbehauptung Goyas damit an, daß er den Anspruch der herkömmlichen Ästhetik auf einen schönen Schein aufgibt und die Zurschaustellung der Dargestellten selbst ins Bild setzt. Deswegen findet er im Nachhinein seine frühen Bilder «unecht», «steif» und «künstlich» (S. 138), weil sie zum Beispiel keine Frauen aus dem Volk zeigen, sondern «Hofdamen, die Majas spielen» (ebd.).

Das Motiv der Maske gewinnt daher im ersten Teil der Romans betrachtlich an Gewicht. Der Künstler meint etwa in bezug auf Don Manuel Godoy: «Es wird nicht einfach sein, Leben in das breite Gesicht des jungen Herzogs zu malen ; denn der repräsentierte gern und legte zu diesem Zweck eine hochmutig blasierte Maske an.» (S. 54) Der Verdienst Goyas bestünde damals darin, den Trug auf dem Gesicht der Dargestellten sichtbar zu machen, den die Zeitgenossen von alleine nicht wahrgenommen hätten. Dies gilt auch für die schöne Lucía Bermúdez, die wiederholt als «zierlich und maskenhaft» (S. 64) oder als «lieblich, dünn, lang, maskenhaft, damenhaft und verrucht» (S. 67) bezeichnet wird und deren Porträt sich als ein Meilenstein von allererster Bedeutung in der erdichteten Laufbahn des Künstlers erweist.

Einen weiteren Schritt geht Goya, wenn er nicht mehr bzw. nicht nur die Maske, sondern das nackte Gesicht hinter jener Maske zu veranschaulichen vermag. Im zweiten Teil des Romans taucht dann immer wieder das Motiv der Nacktheit (besser gesagt: das Wort «nackt» oder «halbnackt») auf, das als das Gegenteil zur Metapher der Maske aufzufassen ist. Diese Gegenüberstellung wird am Projekt des Familiengemäldes, eines der ganz großen Aufträge jener Zeit, überaus deutlich.

Der gutmütige Monarch träumt von einem netten Bild - sprich «was Hübsches, Gemütliches und doch Würdiges» (S.320) -, auf dem er inmitten der Seinigen mit seinen Uhren hantieren oder die Violine spielen würde. Doch Goya will keine Inszenierung nach dem Muster von Van Loos Familie Philipps des Fünften, welches ihm «mit der kunstvoll natürlichen Gruppierung [wie] albernes Theater, ein läppisches Machwerk» (S. 321) vorkommet. Deswegen stellt er mit Einverständnis der gescheiten Königin und zur Verwunderung aller Betrachter die Bourbonen genauso dar, wie sie eben sind, vielleicht sogar etwas zu häßlich. Die Masken sind verschwunden, «nackt und klar die Gesichter» (S. 326).

An diesem Punkt angelangt - der politischen Zwischenstufe seines Reifungsprozesses strebe der geniale Künstler nur noch die Darstellung der - im eigentlichen Sinne des Wortes - bloßen Wirklichkeit an. Seine angebliche Affäre mit der großen Liebe seines Lebens, der Herzogin Cateyana Alba, zwingt ihn aber zur Infragestellung dieser immer noch naiven Vorstellung. Denn im Gegensatz zur schönen falschen Lucía Bermúdez ist die launische Mätresse nicht so leicht zu entlarven, auch wenn es an einer Stelle heißt: «Sie war schön. Ihr Gesicht war nicht maskenhaft, es war das Gesicht einer Liebenden, die kam, ihren Freund in seiner Not zu trösten.» (S. 274)

Der Ausdruck, den sie in jenem Augenblick an den Tag legt, ist zugleich ehrlich und falsch. Die wunderschöne Herzogin sei eigentlich - nach den treffenden Worten ihrer Schwiegermutter - «chatoyante», ein Ausdruck, den Feuchtwanger mit unverhohlenem Vergnügen wiederholt (S. 13, S. 144, S. 296, S. 396) und mit der merkwürdigen, dennoch etymologisch vertretbaren und auf jeden Fall ästhetisch hochbedeutungschwangeren Übersetzung versieht: «Sie schillert wie eine Katze.» (S. 13) Realität. Deswegen führt sie ihren Liebhaber an die Grenze des Demaskierens. Dabei 
geht es nicht schlicht um die «Nacktheit der Gesichter» (S. 373), sondern ums Ganze, da sie - in Feuchtwangers Rekonstruktion durchaus kongruent - die berühmte nackte Maja sei, die höchstpersönlich das unsittliche Bild bei ihrem Verehrer bestelle, nachdem sie ihm die Doña Desnuda des Velásquez ent-hüllt habe. Diesem Wettkampf stelle sich der Künstler nicht nur deswegen, weil «das Verbot der Inquisition, Nacktheit im Bilde darzustellen, [...] eindeutig und streng» (S. 367) sei - also aus politischen Gründen -, sondern auch aus Herausforderung an sich selbt. Was er dabei entdeckt, bedeutet das Ende der Suche nach der nackten Tatsache.

Der einzige Akt in seiner Gesamtproduktion - einer der wenigen in der ganzen spanischen Kunst - lehrt ihn, daß nichts an dieser Frau «echt oder greifbar» (S. 396) ist: "Auch ihre Nacktheit konnte man nicht malen, selbst ihre Nacktheit war verlogen. Und ihr Herz war geschminkt wie ihr Gesicht.» (ebd.) Von diesem Zeitpunkt an werden «die nackten Gesichter» von "großen, schrecklichen Gesichten» abgelöst: «Jetzt spürte er's deutlicher: Das äußere Gesicht mußte ergänzt werden durch die Träume des eigenen Hirns.» (S. 493)

Die Überwindung der klassizistichen Schönheit, der Goya den Kampf angesagt habe, beschränke sich also nicht, wie er zunächst geglaut habe, auf die Enthüllung einer Häßlichkeit, die sich hinter dem schönen Schein verberge. Es gehe nicht lediglich darum, die Masken herunterzureißen, sondern - da selbst die Nacktheit lügen kann darum, die Flatterhaftigkeit des Lebens anzuprangern. Die Fratzen in den Caprichos sind weder Masken noch nackte Gesichter, sie sind beides zugleich: «Die Freunde schauten, und sie sahen, daß diese Gestalten trotz ihrer Maske, durch ihre Masken, nacktere Gesichter hatten als Menschen von Fleisch und Blut.» (S. 581)

Feuchtwangers Romanbiographie deutet also den «argen Weg der Erkenntnis» als eine dreifache Überwindung der klassizistischen Beschönigung der Welt, die von verschiedenen Figuren wie Goyas Schwager Bayeu oder dessen Freund Miguel Bermúdez vertreten wird. Diese Lebensdarstellung, die dem damaligen Stand der Forschung und der romantischen Lektüre von Goyas Æuvre entspricht, bezeugt zweifelsohne eine wenn nicht unbedingt tiefgehende oder avantgardistische, so doch langjährige Beschäftigung mit der Problematik der Wahrheit, der Wirklichkeitswiedergabe, des Realismus in der Kunst. Dabei nimmt die Darstellung der Häßlichkeit einen entscheidenden Platz ein. Nicht nur in Hinsicht auf die alte Infantin und in bezug auf den Maler darf von Faszination die Rede sein. Auch für Feuchtwanger mag sie das wichtigste Moment in Goyas Leistung dargestellt haben, mit dem der Roman nicht von ungefähr schließt.

Beim Betrachten der Bilder scheint er sich immer wieder die Frage gestellt zu haben, wie sich denn die Herrscher, allen voran die Königin, unter solchen Zügen haben darstellen lassen können. Aus diesem Rätsel zieht er auch einen der besten narrativen Effekte des ganzen Romans. Wie oben schon angedeutet, repräsentiert María Luisa ganz herkömmlich die stolze Häßlichkeit. Als der Künstler es wagt, die Bourbonen so zu malen, wie sie tatsächlich aussehen, sind alle Freunde Goyas gespannt auf die Reaktion der Familienmutter. Diese gesteht ganz selbstzufrieden ein: «Das ist ein treues, wahres Bild, geeignet, der Nachwelt zu zeigen, wie wir Bourbonen sind.» (S. 346)

Der Hofmaler stellt dennoch diese adelige Selbstsicherheit auf eine harte Probe, wenn er in der dritten Phase seines Schaffens nicht mehr nur die Gesichter an sich, sondern die innere und vieldeutige Wahrheit ge-stal-ten will, deren die Betroffenen selber sich nicht bewußt sind. In Feuchtwangers Auffassung zeigt nämlich das berühmte Capricho 
Hasta la muerte (Bis zum Tode), auf dem eine alte Frau sich schmückt, die Königin. Sobald Goyas Mätresse, die wunderschöne Cateyana Alba, das Blatt erblickt, versteht sie, worum es geht:

Vor der Uralten, sehr Häßlichen, die in den Spiegel schaut und sich putzt, sagte sie befriedigt: 'Das darfst du sie nicht sehen lassen, Deine Doña María Luisa.' Zu den übrigen Zeichnungen äußerte sie nichts. (S. 570)

Von diesem Zeitpunkt an dauert knapp hundert Seiten lang die Spannung, wie die Königin auf diesen Affront wohl reagieren würde. Die Freundin Goyas, die schöne Pepa, freut sich an diesem Bild (vgl. S. 620). Die nicht minder schöne Lucía Bermúdez sondert es ebenfalls aus der Sammlung aus und rät davon ab, es den Majestäten zu zeigen, denn «alternde Damen sind manchmal empfindlich.» (S. 642) Dennoch läßt der Künstler sich nicht beeindrucken und schenkt die gesamte Reihe dem Königspaar. Am Tag der Übergabe bekommt er selber Angst vor dem eigenen Hoch- und Großmut.

Unter diesen Vorzeichen fängt die Sichtung der Caprichos aus den Augen der Königin an wie eine Sitzung der Inquisition. Amüsiert freut sie sich an den frechen Stichen «ihres» Hofmalers. Entzückt glaubt sie die wunderschöne Alba zu erkennen, die mittlerweile verstorben ist. Und plötzlich entdeckt sie die «sich schmückende, ausgemergelte Uralte» (S. 646):

Sie selber war keineswegs dürr, eher üppig, auch höchstens halb so alt wie diese Uralte. Sie wollte es nicht glauben und wußte es doch sogleich: die äffische, läppische Greisin dieses Blattes, das war sie. Der Atem setzte ihr aus vor dem Insult, dem niederträchtigsten, den sie in ihrem an Kränkungen reichen Leben erlitten hatte. (ebd.)

Feuchtwanger stellt sich vor, was sich in ihrem Geist abspielt. Zum ersten Mal fühlt sie sich in ihrem Stolz gekränkt. Sie weiß natürlich, daß sie nicht schön ist. Sie weiß auch, daß Goya sie nicht zum ersten Mal als häßlich dargestellt hat. Aber sie weiß ebenfalls sofort, daß es diesmal anders ist. Die Leistung Goyas bestünde nicht nur darin, auf jegliche Verschönerung zu verzichten oder gar der Häßlichkeit der Welt ins Gesicht zu sehen, sondern die Realität auf das Wesentlichste zu kondensieren:

Sie war ein Raubvogel und nicht schön, aber einer mit scharfen Augen und guten Krallen, einer, der hoch fliegen konnte und seine Beute rasch erspähen und sicher greifen. Auf diesem Blatt 55 hatte der Mann alles, was an ihr gut war, weggeschwindelt, er hatte nur die Häßlichkeit gemacht und nicht den Stolz, nicht die Kraft. (ebd.)

Dieses Bild verdeutlicht also den Übergang von der zweiten zu der dritten Schaffensphase Goyas: von «auch» der Häßlichkeit bis hin zu «nur» der Häßlichkeit, das heißt den Anspruch auf einen Realismus, der nicht den trügerischen Schein, sondern die Quintessenz der Individuen wiedergibt.

Dies ist genau das, was für die Königin so schwer erträglich war: Daß sie mit ihrer eigenen Ohnmacht, mit dem eigentlichen Grund ihres Charakters konfrontriert wird reduziert auf eine Formel. Bei ihrer Wut gedenkt sie einen Augenblick, den Maler zugrunde zu richten. Aber durch eine Rache würde sie verraten, wie Recht er hatte: Wenn sie ihn der Inquisition auslieferte, dann würde sie für den Rest ihres Lebens diese Schmach tragen müssen, an die man sie auf ewig erinnern würde. Ihre Welt des Protokolls würde zusammenbrechen und sie mit ihr. Deswegen zitiert sie ein angebliches Sprichwort aus ihrer Heimat Parma: «Nur ein Narr ist zornig auf den Spiegel, der sein Bild wiedergibt.» (S. 647) 
ästhetische Urteil der gescheiten María Luisa, die durch die Regeln des Ancien Régime gezwungen wird, die bahnbrechende Neuigkeit der Kunst Goyas zu befürworten, faßt ein Ideal zusammen, das wohl dasjenige Feuchtwangers gewesen sein mag: «Wie diese Alte vor dem Spiegel sitzt, gierig, das ist nicht moralisierend, es ist auch kein wohlfeiler Spaß, es ist die ruhige, traurige, nackte, kahle Wahrheit.» (S. 649).

\section{NOTES}

1. Vgl. Jeannine Baticle, Goya. Paris: Fayard, 1992, S. 258-259: «Les historiens sont divisés quant à l'identité du modèle des majas de Goya; il nous paraît hors de question de suivre les auteurs romantiques qui voulaient y voir sans aucune base documentaire la duchesse d'Albe [...]. En revanche, on ne voit pas pourquoi l'hypothèse selon laquelle les deux toiles de Goya seraient les portraits de Pepita Tudó rencontre si peu d'adhésion [...].»

2. Wir beziehen uns auf folgende Ausgabe: Lion Feuchtwanger, Goya oder Der arge Weg der Erkenntnis. Roman. Berlin und Weimar: Aufbau, 1966. Aus Platzgründen folgt den Zitaten aus diesem Buch allein die Seitenzahl zwischen Klammern. Alle anderen Zitate werden mit Fußnoten versehen.

3. Vgl. Véronique Nahoum-Grappe, «Les canons de la laideur», in Communication n60 (Beauté, Laideur). Paris: Seuil, 1995, S. 29-47, insbesondere S. 29: «L'héroïne d'une histoire d'amour est toujours décrite comme 'belle'.»

4. Trotz eingehender Suche haben wir leider nicht herausfinden können, um welches es sich handelt könnte.

5. Vgl. Wilhelm von Sternburg, Lion Feuchtwanger. Ein deutsches Schriftstellerleben. Frankfurt/Main, Berlin: Ulstein (Lebensbilder), 1987, S. 204: «Die Geschichte der häßlichen Herzogin ist unübersehbar von den Lehren Freuds und Darwins beeinflußt, mit denen sich Feuchtwanger nach dem Ersten Weltkrieg auseinandersetzt. Die physische Konstitution als natürliche Benachteiligung und ihre Auswirkung auf die psychische Entwicklung sind das Kernmotiv des Romans.»

\section{RÉSUMÉS}

Beide Hauptfiguren dieses Künstlerromans, der Maler Francisco de Goya und die Königin María Luisa, sind durch ihre Häßlichkeit gebrandmarkt, die einer Behinderung gleichkommt und sie den Lehren von Darwin und Freud entsprechend zur Selbstüberwindung zwingt. Die Infragestellung der Schönheit beschränkt sich aber hier nicht auf diese im realistischen Roman herkömmliche Gestaltung der Figuren. Wichtiger ist Feuchtwangers Interpretation von Goyas Schaffen als Überwindung der klassizistischen Ästhetik : Die Leistung des Malers bestünde nicht nur darin, daß er nicht alles verschönert oder die Welt so darlegt, wie sie eben aussieht, sondern daß er die innere Wahrheit zu zeigen vermag, indem er den Charakter der Dargestellten 
schonungslos auf das Wesentlichste reduziert und zum Beispiel im berühmten Bild « Hasta la muerte» lediglich die Häßlichkeit der Königin zur Schau stellt. Somit äußert der Schriftsteller ein Ideal, das sein eigenes gewesen sein mag.

Les deux personnages principaux de cette biographie romancée, le peintre Francisco de Goya et la reine María Luisa, se distinguent par leur laideur, qui équivaut à un handicap et qui, conformément aux leçons de Darwin et de Freud, les obligent à se dépasser eux-mêmes. Cependant, la remise en question de la beauté ne se limite ici à cette conception traditionnelle des personnages dans le roman réaliste. Le plus important est l'interprétation que Feuchtwanger fait de l'œuvre de Goya conçue comme un dépassement de l'esthétique classique. Le mérite du peintre ne consisterait pas seulement à ne pas tout embellir ou à représenter le monde tel qu'il a l'air d'être, mais à savoir montrer la vérité intérieure en ramenant sans ménagements le caractère des personnages à l'essentiel et, par exemple dans la célèbre gravure « Hasta la muerte ", en n'exhibant que la seule laideur de la reine. De cette manière, le romancier formule un idéal qui pourrait bien être le sien.

INDEX

Mots-clés : peinture, beauté, laideur

\section{AUTEURS}

\section{FRÉDÉRIC WEINMANN}

Lycée Watteau (Valenciennes) 